\title{
ESTUDO DA CONVERSÃO CATALÍTICA DE GLICOSE EM CATALISADOR HETEROGÊNEO DE TITÂNIA NANO-ANATÁSIO
}

\author{
G. P. NOGUEIRA ${ }^{1}$, C. A. S. LANZIANO ${ }^{1}$, R. GUIRARDELLO ${ }^{1}$, C. B. RODELLA ${ }^{2}$ \\ ${ }^{1}$ Universidade Estadual de Campinas, Faculdade de Engenharia Química \\ ${ }^{2}$ Centro Nacional de Pesquisa em Energia e Materiais, Laboratório Nacional de Luz \\ Síncrotron \\ E-mail para contato: gui_pessoan@hotmail.com
}

\begin{abstract}
RESUMO - O crescente interesse pelo beneficiamento da biomassa tornou-se tanto de caráter científico quanto econômico. Originando-se da hidrólise celulósica, a glicose se mostra uma matéria prima abundante, podendo originar uma gama de produtos de alto interesse econômico, dentre eles a frutose e o HMF. Um meio para isto é através da catálise heterogênea com óxidos como titânia, zircônia e alumina. $\mathrm{O}$ uso de titânia com conformação em fase anatásio mostrou bons resultados em termos de conversão e seletividade. Para tanto foi testado o desempenho da titânia nano-anatásio na conversão de glicose, realizando posteriormente um ajuste numérico para estimativa de parâmetros no modelo cinético de reações proposto.
\end{abstract}

\section{INTRODUÇÃO}

A busca por meios alternativos ao petróleo para a obtenção de energia e materiais renováveis é de interesse mundial, e figura dentre as áreas de pesquisa mais importantes do Brasil. A produção de biocombustíveis é crescente desde a década passada no país, tanto o etanol como biodiesel. Neste cenário, portanto, a biomassa se torna um componente chave para a geração de produtos sustentáveis, sejam estes combustíveis de segunda geração ou potenciais monômeros como o HMF (hidroximetil furfural) (RODRIGUES, 2011; SOUZA et al., 2012).

A biomassa celulósica é composta principalmente por carboidratos, lignina, proteínas e gorduras. Sendo a celulose um dos principais componentes, sendo possível a sua hidrólise para a obtenção da glicose. Esta, por sua vez, consiste no ponto inicial para a obtenção de diversos produtos como o etanol, HMF, acido levulínico, dentre outros (LANZIANO, 2014; SOUZA et al., 2012).

O estudo da isomerização da glicose em frutose data de 1895 pelos pesquisadores Lobry de Bruyn e Alberta Van Ekenstein, onde estudavam a reação em meios básicos por catálise alcalina. Com o tempo, observou-se também o ocorrência desta também em meios ácidos e neutros. Catalisadores ácidos de Lewis, em especial, apresentam maior afinidade no processo de isomerização, polarizando o grupo carbonila da cetona e promovendo a 
transferência do grupo hidroxila do carbono 1 para 2 (LANZIANO, 2014; ROMÁNLESHKOV et al., 2010).

O processo de isomerização da glicose já é realidade, sendo um dos mariores processos industriais bioquímicos da atualidade. Através da atividade da catalítica da enzima xilose isomerase, atinge-se equilíbrios químicos de $42 \%$ frutose e $50 \%$ glicose (frações mássicas). Porém também apresenta diversos empecilhos, tais como grande uso de soluções tampão para controle do $\mathrm{pH}$, temperatura de operação limitada, reposição periódica de enzimas irreversívelmente desativadas. Características comuns de processos com catálise enzimática. Deste modo, a busca por outros catalisadores se torna uma necessidade para redução de custos e aumento de produtividade (ROMÁN-LESHKOV et al., 2010; SOUZA et al., 2012).

O estudo do desempenho de catalisadores heterogêneos com características ácidas para este fim ganhou interesse. Visto que possuem sítios ácidos de Lewis e de Bronsted ao longo de sua superfície mesmo em meio aquoso. Assim, catalisadores como o óxido de titânio e de zircônio apresentaram elevado potencial para a conversão da glicose em frutose e outros produtos de interesse como o HMF e ácido levulínico (WATANABE et al., 2005; LANZIANO, 2014).

A utilização de um catalisador heterogêneo acarreta cuidados com área superficial, conformação da cadeia, densidade de sítios básicos e ácidos e seu tipo, visto que estas características afetam diretamente no mecanismo de reação, e portanto nos parâmetros cinéticos estudados (WATANABE et al., 2005).

\section{METODOLOGIA}

\subsection{Preparo do catalisador}

O catalisador óxido de titânio fase anatásio foi obtido via síntese hidrotérmica. Deste modo, foram mantidos $200 \mathrm{~mL}$ de água deionizada em banho de gelo e adicionou-se lentamente $9 \mathrm{~mL}$ de tetracloreto de titânio sob agitação constante. Após, foram adicionados 17,3 g de ácido cítrico sob agitação. Acoplou-se então a mistura a um condensador vertical, imergindo a mesma em um banho de óleo sob aquecimento. A mistura foi mantida sob agitação e a temperatura de $120^{\circ} \mathrm{C}$ durante $16 \mathrm{~h}$. A mistura foi então centrifugada e lavada com água deionizada a temperatura ambiente repetidas vezes de modo a retirar resíduos ácidos do catalisador. Por fim, o óxido resultante foi levado à estufa por $16 \mathrm{~h}$ a $120^{\circ} \mathrm{C}$ para retirada de água residual.

\subsection{Ensaios cinéticos}

Os ensaios cinéticos foram realizados em um reator do tipo batelada de liga HASTELLOY de $300 \mathrm{~mL}$. O reator possui um revestimento interno removível de politetraflouretileno $\left(\right.$ Teflon $^{\circledR}$ ) de modo a minimizar a influência da superfície metálica sobre a reação. Foram misturados $160 \mathrm{~mL}$ de solução aquosa de glicose $2 \%$ (fração mássica) com 2,666 g de catalisador e inseridos no reator. O reator foi então acoplado a um sistema de agitação e controle de pressão e temperatura. 
Inicialmente, é realizada uma purga da atmosfera interna do reator com gás nitrogênio para minimizar sua influência sobre a cinética reacional. A agitação foi mantida a $100 \mathrm{rpm}$ até a temperatura do sistema atingir à de reação, após foi mantida a $500 \mathrm{rpm}$ até o final do ensaio. O tempo de reação foi de 7 horas à temperatura de $120^{\circ} \mathrm{C}$, com a retirada de 8 alíquotas em tempos determinados por meio de um registro acoplado ao reator. Cada alíquota foi centrifugada para remoção do catalisador, resfriada e posteriormente enviada para análise cromatográfica por HPLC (cromatografia líquida de alta precisão).

Tabela 1 - Distribuição das alíquotas ao longo do tempo de reação.

\begin{tabular}{|l|cccccccc|}
\hline Alíquota & $\mathrm{A} 1$ & $\mathrm{~A} 2$ & $\mathrm{~A} 3$ & $\mathrm{~A} 4$ & $\mathrm{~A} 5$ & $\mathrm{~A} 6$ & $\mathrm{~A} 7$ & $\mathrm{~A} 8$ \\
\hline Tempo & 0 & $30 \mathrm{~min}$ & $1 \mathrm{~h}$ & $1 \mathrm{~h} 30 \mathrm{~min}$ & $2 \mathrm{~h}$ & $3 \mathrm{~h}$ & $5 \mathrm{~h}$ & $7 \mathrm{~h}$ \\
\hline
\end{tabular}

\subsection{Modelo cinético e ajuste de parâmetros}

O modelo abaixo descrito foi proposto por LANZIANO (2014) para descrever a cadeia de reações envolvendo a conversão de glicose nas dadas condições experimentais. $\mathrm{O}$ modelo leva em conta principalmente a relação da formação de produtos com sítios ácidos e básicos de catalisadores heterogêneos titânia e zircônia puros e mistos entre si. Uma vez que análises por TPD (dessorção à temperatura programada) demonstraram um número muito maior de sítios ácidos em relação aos básicos, desconsiderou-se estes para simplificação do modelo.

Figura 1 - Modelo cinético simplificado de conversão da glicose (LANZIANO, 2014).

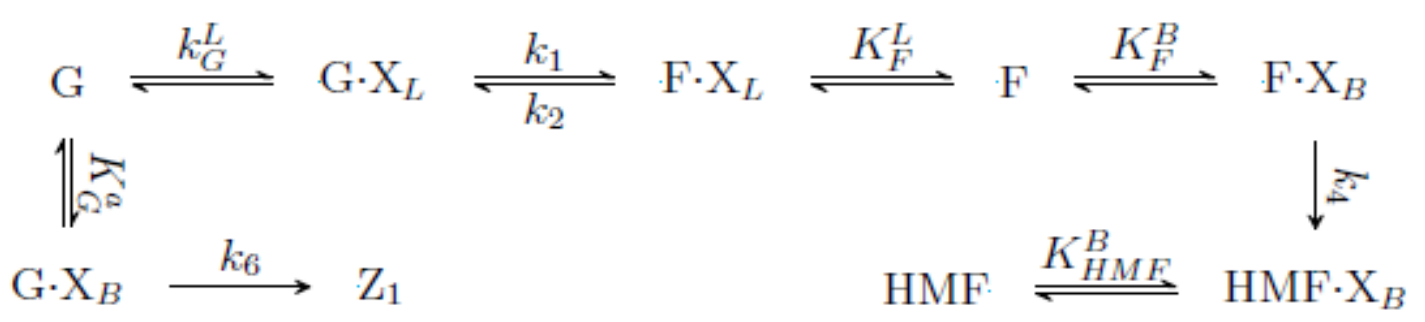

Este, por sua vez, descreve a interação da glicose e seus produtos com sítios ácidos de Bronsted e Lewis por meio de adsorção e dessorção associados à reações de conversão na superfície do catalisador. $\mathrm{X}_{\mathrm{L}}$ e $\mathrm{X}_{\mathrm{B}}$ representam respectivamente os sítios ácidos de Lewis e de Bronsted, logo estão associados ao modelo cinético parâmetros de transporte de massa de adsorção e dessorção, representados com L e G para os ambos os tipos de sítios.

Deste modo, uma molécula de glicose $(\mathrm{G})$ em solução pode adsorver em um sítio ácido de Lewis e em seguida converter-se em frutose $(\mathrm{F})$ ou dessorver-se. A frutose por sua vez pode adsorver-se em um sítio ácido de Bronsted e coverter-se em HMF ou dessorver-se. E assim por diante com o HMF e produtos indeterminados $Z_{1}$.

Uma vez que os ensaios cinéticos foram realizados em batelada, pode-se aplicar um modelo diferencial para descrever os mesmos. O balanço de concentração das espécies pode ser simplificado associando a concentração das espécies adsorvidas com as em solução 
através da suposição de equilíbrio entre as etapas de adsorção e dessorção das mesmas. Sob esta ótica, também é possível quantificar a quantia de sítios ácidos livres através da subtração do número de sítios totais medido experimentalmente pelo número de sítios ocupados. Considerando as constantes de equilíbrio de adsorção para a glicose e a frutose são menores que a unidade, pode-se aproximar a quantia de sítios ácidos livres para um valor médio. Deste modo, o modelo é descrito pelas equações 1 a 6 .

$$
\begin{aligned}
& \frac{d[G]}{d t}=k_{2}^{\prime}[F]\left[X_{L}\right]-k_{1}^{\prime}[G]\left[X_{L}\right]-k_{6}^{\prime}[G]\left[X_{B}\right] \\
& \frac{d[F]}{d t}=k_{1}^{\prime}[G]\left[X_{L}\right]-k_{2}^{\prime}[F]\left[X_{L}\right]-k_{4}^{\prime}[F]\left[X_{B}\right] \\
& \frac{d[H M F]}{d t}=k_{4}^{\prime}[F]\left[X_{B}\right] \\
& {\left[X_{B}\right]=\frac{\left[X_{B}\right]_{0}}{1+K_{H M F}^{B}[H M F]}} \\
& {\left[X_{L}\right]=0,9\left[X_{L}\right]_{0}} \\
& k_{1}^{\prime}=k_{1} K_{G}^{L} \quad k_{2}^{\prime}=k_{2} K_{F}^{L} \quad k_{4}^{\prime}=k_{4} K_{F}^{B} \quad k_{6}^{\prime}=k_{6} K_{G}^{B}
\end{aligned}
$$

Realizando uma primeira estimativa dos parâmetros presentes em $\mathbf{p}$ e q, é possível resolver o sistema de equações diferenciais ordinárias do modelo a partir do método de Runge-Kutta de $4^{\mathrm{a}}$ ordem. Uma vez resolvido, aplica-se o método da máxima verossimilhança de modo a minimizar a diferença entre os dados experimentais $\tilde{\boldsymbol{\eta}}$ e os dados obtidos a partir da solução do sistema de EDO's. Onde m é denominada função objetivo e w representa a variância dos dados experimentais. Deste modo, minimizou-se a função objetivo a partir da variação dos parâmetros em p e q com a utilização do solver do software Microsoft Office Excel $2007^{\circledR}$.

$$
\begin{aligned}
& p=\left(k_{1}^{\prime}, k_{2}^{\prime}, k_{4}^{\prime}, k_{6}^{\prime}\right) \\
& q=\left(K_{B}^{H M F}\right) \\
& m(p, q)=\sum \frac{1}{w}(\tilde{\eta}-\eta)^{2}
\end{aligned}
$$

\section{RESULTADOS E DISCUSSÃO}

Na Tabela 2 encontram-se os resultados da análise por HPLC das alíquotas provenientes do ensaio catalítico realizado. $\mathrm{O}$ modelo descrito anteriormente foi realizado com base nestes resultados, desconsiderando espécies de baixa concentração.

Tabela 2 - Análise por HPLC das alíquotas retiradas 


\begin{tabular}{|c|c|c|c|c|c|c|c|}
\hline & $\begin{array}{c}\text { Ác. Fórmico } \\
\mathbf{g} / \mathbf{L}\end{array}$ & $\begin{array}{c}\text { Ác. Láctico } \\
\mathbf{g} / \mathbf{L}\end{array}$ & $\begin{array}{c}\text { Ác. } \\
\text { Levulínico }\end{array}$ & $\begin{array}{c}\text { Frutose } \\
\mathbf{g} / \mathbf{L}\end{array}$ & $\begin{array}{c}\text { Glicose } \\
\mathbf{g} / \mathbf{L}\end{array}$ & HMF g/L & Furfural g/L \\
\hline A1 & 0,0000 & 0,0000 & 0,2957 & 0,0000 & 20,6908 & 0,0380 & 0,0005 \\
A2 & 0,0000 & 0,0000 & 0,0622 & 0,7210 & 18,4847 & 0,3873 & 0,0048 \\
A3 & 0,0000 & 0,0000 & 0,0595 & 0,8810 & 17,0461 & 0,7484 & 0,0080 \\
A4 & 0,0000 & 0,0000 & 0,0507 & 0,9010 & 16,3023 & 1,2051 & 0,0102 \\
A5 & 0,0000 & 0,0000 & 0,0545 & 0,8810 & 15,5554 & 1,3192 & 0,0128 \\
A6 & 0,0000 & 0,0000 & 0,1230 & 0,8230 & 14,3927 & 1,7819 & 0,0169 \\
A7 & 0,0000 & 0,0000 & 0,0590 & 0,6360 & 11,1338 & 1,6886 & 0,0239 \\
A8 & 0,0000 & 0,0000 & 0,0923 & 0,6220 & 11,0701 & 2,7516 & 0,0287 \\
\hline
\end{tabular}

Para o ajuste é necessária a determinação dos sítios ácidos totais de Bronsted e Lewis, ou seja, no instante inicial da reação. Para isto, utilizou-se os resultados de análise por TPD obtidos por LANZIANO (2014) para o catalisador titânia em fase anatásio de $630 \mu \mathrm{mol} / \mathrm{g}$. Sendo a proporção de sítios ácidos de Bronsted e Lewis de 3:1 respectivamente. Com os dados de 2,666 g de catalisador por reação e volume de líquido de $160 \mathrm{~mL}$, obtém-se o valor total de sítios ácidos.

Deste modo aplicou-se o método de Runge-Kutta de $4^{\mathrm{a}}$ ordem ao sistema de EDO's acima, resolvendo as equações simultâneamente ao ajuste dos parâmetros cinéticos. Cujos valores encontram-se na Tabela 3, com sua respectiva representação gráfica na Figura 2. Todos os parâmetros encontram-se em L.mol ${ }^{-1} \cdot \mathrm{min}^{-1}$, com exceção de $\mathrm{K}_{\mathrm{HMF}}{ }^{\mathrm{B}} \mathrm{em} \mathrm{L}$. $\mathrm{mol}^{-1}$.

Tabela 3 - Ajuste dos parâmetros cinéticos.

\begin{tabular}{|lr|}
\hline \multicolumn{2}{|c|}{ Parâmetros encontrados } \\
\hline k1' & 1,068229586 \\
k2' & 7,832771706 \\
k4' & 4,545663293 \\
k6' & 0,256560227 \\
KBHMF & 40,63545861 \\
\hline
\end{tabular}

Tabela 4 - Coeficientes de regressão.

\begin{tabular}{|ccl|}
\hline \multicolumn{3}{|c|}{ Coeficiente de Regressão $\left(R^{2}\right)$} \\
\hline Glicose & Frutose & HMF \\
0,967324433 & 0,952639311 & 0,797104753 \\
\hline
\end{tabular}

Observa-se que o ajuste possui menor precisão quanto ao HMF, o que pode ser explicado por erros experimentais tanto no processo de retirada de alíquotas como na seção de análise, evidenciado por pontos de comportamento inesperado, como em 300 minutos. Porém, mesmo não ocorrendo um bom ajuste, é possível observar uma tendência de comportamento clara a partir dos pontos experimentais na Figura 2.

Figura 2 - Concentração das espécies por tempo. 


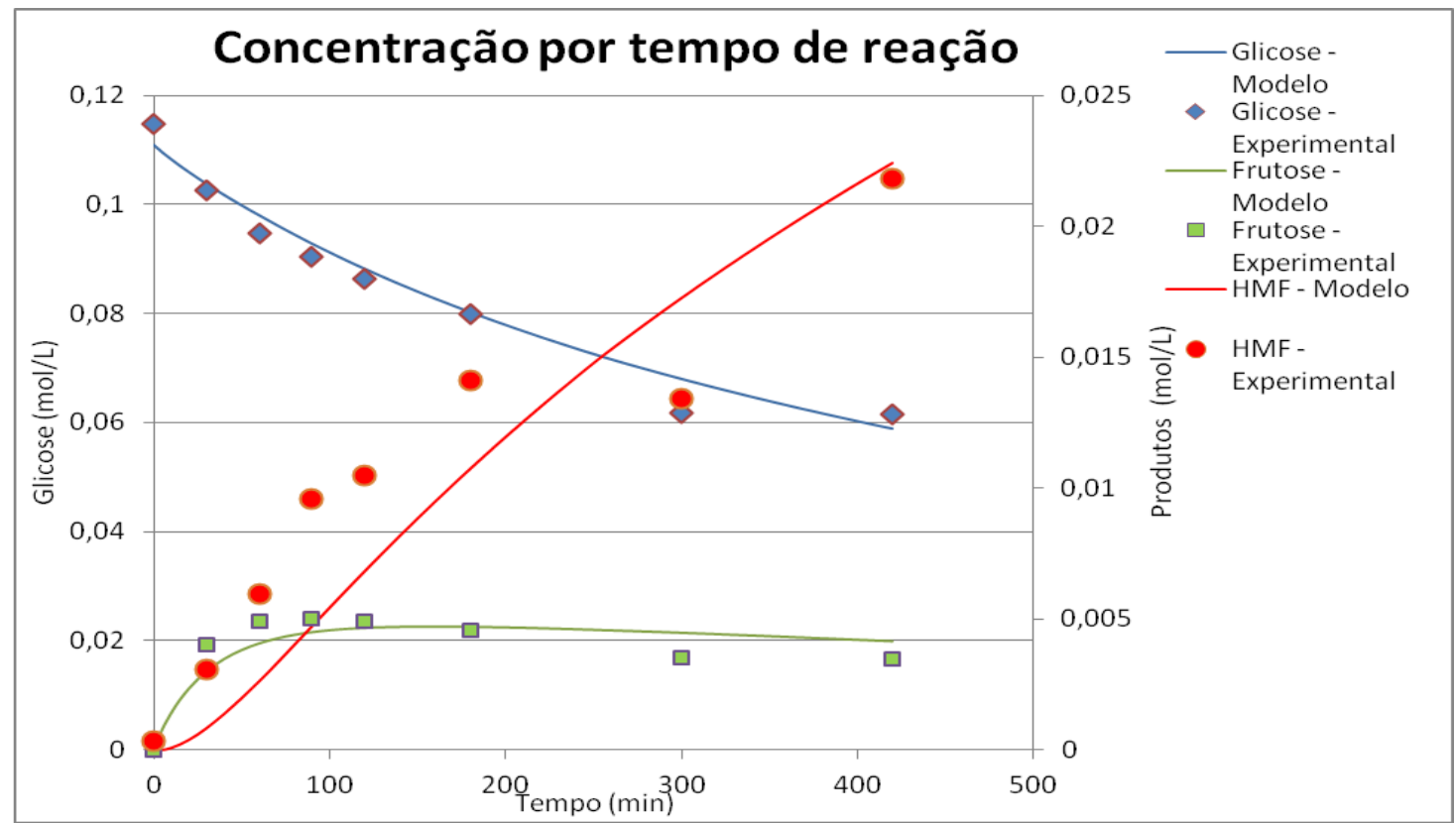

\section{CONCLUSÃO}

Uma vez que o catalisador utilizado, sintetizado em forma de nanopartículas, possui características estruturais diferentes em relação ao utilizado por LANZIANO (2014), não é possível concluir quanto à precisão do ajuste. Maiores análises de quantificação de sítios ácidos e sua proporção na superfície do catalisador são essenciais tanto para a caracterização deste quanto para a validação do modelo aplicado.

\section{REFERÊNCIAS}

LANZIANO, C.A.S. Avaliação de catalisadores mistos de titânia - zircônia na reação de isomerização da glicose a frutose e ajuste de parâmetros da cinética de reação. Dissertação (mestrado). Universidade Estadual de Campinas, Faculdade de Engenharia Química. 2014.

NAKAJIMA, K.; NOMA, R.; KITANO, M.; HARA,M. Selective glucose transformation by titania as a heterogeneous Lewis acid catalyst. Journal of Molecular Catalysis A: Chemical. 2013.

RODRIGUES, J.A.R. Do engenho à biorrefinaria. a usina de açúcar como empreendimento industrial para a geração de produtos bioquímicos e biocombustíveis. Quim. Nova. 2011.

ROMÁN-LESHKOV, Y.; MOLINER, M.; LABINGER, J.A.; DAVIS, M.E. Mechanism of glucose isomerization using a solid lewis acid catalyst in water. Angew. Chem. Int. Ed. 2010.

SOUZA, R.O.L.; FABIANO, D.P.; FECHE, C. Glucose-fructose isomerisation promoted by basic hybrid catalysts. Catalysis Today. 2012. 